\title{
Pneumocyte Biomarkers KL-6 and Surfactant Protein D Reflect the Distinct Findings of High-Resolution Computed Tomography in Nonspecific Interstitial Pneumonia
}

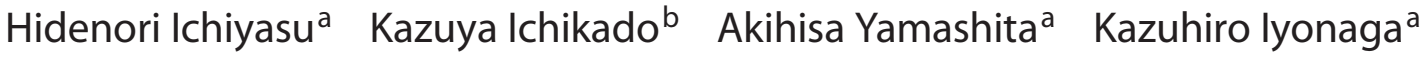 \\ Osamu Sakamoto $^{a}$ Moritaka Suga $^{b}$ Hirotsugu Kohrogi ${ }^{a}$ \\ ${ }^{a}$ Department of Respiratory Medicine, Graduate School of Medical Sciences, Kumamoto University, and \\ ${ }^{b}$ Division of Respiratory Medicine, Saiseikai Kumamoto Hospital, Kumamoto, Japan
}

\section{Key Words}

High-resolution computed tomography $\cdot \mathrm{KL}-6$ •

Nonspecific interstitial pneumonia $\cdot$ SP-D

\begin{abstract}
Background: Serum levels of pneumocyte biomarkers KL- 6 and surfactant protein D (SP-D) are useful diagnostic markers for interstitial lung diseases. However, associations of serum KL- 6 and SP-D with radiologic findings in nonspecific interstitial pneumonia (NSIP) remain unclear. Objectives: To determine whether serum levels of KL- 6 and SP-D reflect fibrotic and/or inflammatory processes in NSIP, we investigated the correlation between high-resolution computed tomography (HRCT) findings and serum KL- 6 and SP-D levels. Methods: Serum levels of KL- 6 and SP-D were measured in 21 patients with biopsy-confirmed NSIP. The radiographic extent of 6 HRCT patterns and total HRCT score, defined as the scored fibrotic index, were assessed. Changes in the levels of serum markers and CT findings during follow-up were also monitored. Results: Serum levels of KL- 6 in NSIP positively correlated with the total HRCT score and overall extent of interstitial disease. Serum levels of SP-D in NSIP showed a positive correlation with the area of ground-glass attenuation without traction bronchiectasis and the inflammatory CT pattern, but the levels were inversely correlated with the area of ground-glass attenuation with traction bronchiecta-
\end{abstract}

sis and the fibrotic CT pattern. The follow-up CT and serum marker changes after treatment showed that percent change of disease extent was reflected in both markers, especially $\mathrm{KL}-6$. Further, the decreased fibrotic pattern correlated with both biomarkers. Conclusions: The results indicate that serum levels of KL- 6 in NSIP reflect the overall extent of interstitial lesions, which include both inflammatory and fibrotic lesions, while the levels of SP-D mainly reflect the extent of inflammatory lesions.

Copyright $\odot 2011$ S. Karger AG, Basel

\section{Introduction}

Idiopathic interstitial pneumonias (IIPs), a type of diffuse parenchymal lung disease, are characterized by different patterns of intra-alveolar fibrosis and alveolitis. Idiopathic pulmonary fibrosis (IPF) and nonspecific interstitial pneumonia (NSIP) are the main types of IIPs [1]. Response to treatment and the prognosis of these diseases are distinct: poor response and progressive deterioration of lung function and short median survival are observed in IPF, whereas better response and prognosis are observed in NSIP [1-6]. Katzenstein and Fiorelli [7] divided NSIP into 3 subgroups on the basis of the extent of inflammation and/or fibrosis observed in biopsy specimens. NSIP patients with predominant inflammation

\section{KARGER}

Fax +41613061234 E-Mail karger@karger.ch www.karger.com
(C) 2011 S. Karger AG, Basel

$0025-7931 / 12 / 0833-0190 \$ 38.00 / 0$

Accessible online at:

www.karger.com/res
Hidenori Ichiyasu, MD, PhD

Department of Respiratory Medicine, Graduate School of Medical Sciences

Kumamoto University, 1-1-1 Honjo

Kumamoto 860-8556 (Japan)

Tel. +81 96373 5012, E-Mail ichiyasu@ kumamoto-u.ac.jp 
Table 1. Baseline characteristics of the study patients

\begin{tabular}{lc}
\hline & NSIP \\
\hline Patients, $\mathrm{n}$ & 21 \\
$\quad$ Male & 8 \\
$\quad$ Female & 13 \\
Age, years & $49.8 \pm 13.3$ \\
Smoking history & \\
$\quad$ Never smokers & 11 \\
Ex-smokers & 3 \\
Current smokers & 7 \\
Histological diagnosis & 3 \\
$\quad$ Cellular & 18 \\
Fibrotic & \\
Pulmonary function test & $71.9 \pm 16.5$ \\
$\quad$ VC, \% of predicted & $81.4 \pm 5.8$ \\
FEV $_{1}, \%$ & $52.6 \pm 14.6$ \\
DLCO, \% of predicted $_{\text {PaO }}$, mm Hg & $84.2 \pm 7.8$ \\
PaCO $_{2}$, mm Hg & $40.9 \pm 2.9$ \\
\hline
\end{tabular}

Means \pm SD, unless indicated otherwise. $V C=$ Vital capacity; $\mathrm{FEV}_{1}=$ forced expiratory volume in $1 \mathrm{~s} ;$ DLCO = diffusion capacity of the lung for carbon monoxide; $\mathrm{PaO}_{2}=$ arterial oxygen tension; $\mathrm{PaCO}_{2}=$ carbon dioxide arterial tension.

have a considerably better prognosis than those with predominant fibrosis.

IIPs are frequently diagnosed on the basis of the distinct patterns observed in a high-resolution computed tomography (HRCT) scan. Several studies suggested that the HRCT findings and pathological findings showed good correlation in IIPs [8-11]. The relative extents of ground-glass attenuation and honeycombing appear to be the most important imaging features of CT facilitating the differentiation between NSIP and IPF. The American Thoracic Society and the European Respiratory Society have jointly released the international multidisciplinary consensus statement that recommends that diagnoses should be made on the basis of the correlation of clinicoradiological-pathological features [1]. Johkoh et al. [10] compared the CT findings and histological findings of NSIP and reported that the extent of traction bronchiectasis on HRCT was associated with increased fibrosis.

The serum levels of KL- 6 and surfactant protein-D (SP-D), which are lung-specific proteins, have been shown to be correlated with clinical manifestations such as the extent and activity of pulmonary fibrosis and inflammation [12-17]. KL-6 is a circulating glycoprotein and is classified as MUC1 mucin. Kohno et al. $[12,18]$ and Hirasawa et al. [19] found that KL-6 is a chemotactic factor for human lung fibroblasts; further, they reported that the serum levels of KL- 6 reflected the disease status of various interstitial pneumonias such as IPF, hypersensitivity pneumonia, and radiation pneumonia. Meanwhile, Takahashi et al. [13] demonstrated that the serum levels of SP-D in patients with IPF might be useful not only for the diagnosis of IPF but also for the estimation of their clinical response to treatment and prognosis.

Although some studies have investigated the relationship between HRCT findings and serum markers or pulmonary functions in IPF patients, the possible associations between HRCT findings and the levels of KL-6 and SP-D in NSIP patients have not yet been investigated completely. In biopsy-proven NSIP, we categorized 6 CT findings: (1) normal lung attenuation, (2) ground-glass attenuation without traction bronchiectasis, (3) airspace consolidation without traction bronchiectasis, (4) ground-glass attenuation with traction bronchiectasis, (5) airspace consolidation with traction bronchiectasis, and (6) honeycombing. To determine the role of serum markers in evaluating disease severity, we compared the serum levels of KL-6 and SP-D with these HRCT findings of NSIP patients.

\section{Methods}

\section{Study Population}

We retrospectively studied patients with surgical lung biopsyproven NSIP. The histological specimens were reviewed by an independent expert pulmonary pathologist. The diagnosis of idiopathic NSIP was based on history, physical examination, HRCT, and histological examination, in accordance with the American Thoracic Society/European Respiratory Society consensus classification $[1,5]$. The baseline characteristics of 21 NSIP patients, including demographics, pathological NSIP pattern, and lung function, are summarized in table 1 . This study was approved by the ethics committee at our institution, and a written informed consent was obtained from all the patients. All patients underwent treatment with corticosteroids, with or without immunosuppressive agents, between the initial and follow-up CT scans. The mean duration was 5.4 months (4-12 months).

Measurement of the Levels of Serum Markers

Peripheral blood samples were collected from the patients at their initial visits or before lung biopsy, and used for KL-6 and SP-D assays. The serum level of KL-6 (normal $<500 \mathrm{U} / \mathrm{ml}$ ) was measured by performing an enzyme-linked immunosorbent assay (ELISA) using the EITEST KL-6 kit (Eisai, Tokyo, Japan). SP-D level (normal $<110 \mathrm{ng} / \mathrm{ml}$ ) was measured using a commercial ELISA kit (Yamasa, Tokyo, Japan). All the assays were performed in duplicate, and the data were expressed as mean values.

\section{CT Scanning Protocol}

All patients had HRCT studies of the thorax. Collimation was at 1-2 $\mathrm{mm}$ with the images acquired at $1-$ or $2-\mathrm{cm}$ intervals. All 
images were reconstituted with a high-spatial-frequency algorithm using a previously reported method [20]. The average time between HRCT imaging and biopsy was 2 weeks.

\section{Radiologic Evaluation}

All of the CT images were reviewed by two observers (H.I. and K.I.) who were specialists for CT imaging and working together in the same medical team, and final decisions on the extent of each CT finding were reached by consensus without the knowledge of clinical information. The observers assessed the presence and the extent of ground-glass attenuation, airspace consolidation, traction bronchiectasis, and honeycombing, and their different combinations (table 2). These 6 types of HRCT findings were defined as described previously [10]. The overall extent of interstitial disease was estimated on the basis of the area of increased lung attenuation, which did not include the area of normal attenuation.

\section{Scoring of HRCT Findings}

The HRCT findings were graded on a scale of $1-6$ on the basis of the classification reported by Ichikado et al. [20] (table 2). The lungs were divided into 6 zones (each lung was divided into an upper, a middle, and a lower side), and each zone was evaluated separately. The extent of the involvement of each finding was assessed visually for each lung zone and was estimated to the nearest $10 \%$ of parenchymal involvement. The overall percentage of the involvement of 1 type of abnormal finding was obtained by averaging the values of this finding of all the 6 lung zones; this final value was defined as disease extent (in \%; tables 2, 3). The total HRCT score, defined as the fibrotic index score, was calculated by multiplying the disease extent value by each corresponding score and summing up all these grading scores. Table 2 presents the disease extent values, the CT grading scores, and the total HRCT score of a representative case.

Association between the Total HRCT Score, the Overall Extent of Interstitial Disease, the Disease Extent of the 6 Types of

HRCT Findings, and the Serum Levels of KL-6 and SP-D

The total HRCT score was calculated, and the associations between the score and serum levels of KL-6 and SP-D were analyzed. Furthermore, the overall extent of interstitial disease and each of the 6 types of HRCT findings were also analyzed to determine the correlation of these findings with the serum levels of KL-6 and SP-D.

Association between the HRCT Patterns (Inflammatory or

Fibrotic) and the Serum Levels of KL- 6 and SP-D

On the basis of the absence or presence of traction bronchiectasis, HRCT findings were divided into 2 patterns: inflammatory and fibrotic. The inflammatory pattern was defined by the absence of traction bronchiectasis on ground-glass attenuation and airspace consolidation, and the fibrotic pattern was defined by the presence of traction bronchiectasis on ground-glass attenuation and airspace consolidation, and honeycombing. Correlations between the extent of the 2 HRCT patterns and the serum levels of KL- 6 and SP-D were also determined.

Changes in the Extent of HRCT Findings and Their Associations with the Serum Levels of KL- 6 and SP-D before and after Treatment

Finally, changes in the levels of the serum markers, the extent of disease, and the total HRCT score before and after treatment
Table 2. HRCT findings, corresponding score, and HRCT scores of a representative case

\begin{tabular}{lllc}
\hline HRCT findings & Score & $\begin{array}{l}\text { Disease } \\
\text { extent, \% }\end{array}$ & $\begin{array}{l}\text { HRCT } \\
\text { score }\end{array}$ \\
\hline $\begin{array}{l}\text { Normal attenuation } \\
\begin{array}{l}\text { Ground-glass attenuation without } \\
\text { traction bronchiectasis }\end{array}\end{array}$ & 1 & 76.7 & 76.7 \\
$\begin{array}{l}\text { Airspace consolidation without } \\
\quad \text { traction bronchiectasis }\end{array}$ & 3 & 13.3 & 26.6 \\
$\begin{array}{l}\text { Ground-glass attenuation with } \\
\text { traction bronchiectasis }\end{array}$ & 4 & 5.0 & 0 \\
$\begin{array}{l}\text { Airspace consolidation with } \\
\text { traction bronchiectasis }\end{array}$ & 5 & 5.0 & 20.0 \\
$\begin{array}{l}\text { Honeycombing } \\
\text { Total HRCT score }\end{array}$ & 6 & 0 & 0 \\
\hline
\end{tabular}

Disease extent (\%) was defined as the percentage of 1 type of HRCT finding obtained by averaging the values obtained for 6 lung zones. The HRCT score was calculated by multiplying the disease extent value by each corresponding score. The total HRCT score was calculated by summing up each grading HRCT score, as described in the Methods section.

were expressed in terms of percent change from the baseline. The correlation between these variables was also analyzed.

Statistical Analysis

The results were expressed as means $\pm \mathrm{SD}$. The differences between the variables were assessed by the Mann-Whitney U test. Spearman's rank correlation test (coefficient of correlation, $r_{S}$ ) was used to assess associations between the HRCT scores, the overall extent of the interstitial disease, the extent of the 6 HRCT findings, the HRCT patterns, and the serum levels of KL- 6 or SP-D. Statistical analysis was performed using StatView-J 5.0 software (Abacus Concept, Berkeley, Calif., USA). The difference was considered to be statistically significant (two-tailed tests) if $\mathrm{p}<0.05$.

\section{Results}

\section{Associations between the Total HRCT Score and the Serum Levels of KL- 6 and SP-D}

The HRCT findings from 21 patients with NSIP were evaluated for assessing the correlation between the total HRCT score and the mean serum levels of KL-6 (2,045.2 $\pm 1,333.8 \mathrm{U} / \mathrm{ml})$ and SP-D (352.6 $\pm 203.6 \mathrm{ng} / \mathrm{ml})$. The mean total HRCT scores ranged from 146.8 to 261.4 points (192.1 \pm 33.8 points). Spearman's rank correlation test revealed a significant correlation between the HRCT score and the KL-6 levels $\left(r_{S}=0.453 ; p<0.05\right)$. However, no correlation was observed between the HRCT score and the SP-D levels (fig. 1). In fibrotic NSIP patients $(n=18)$, 
Table 3. Correlation between the extent of HRCT findings and serum markers

\begin{tabular}{|c|c|c|c|c|c|}
\hline \multirow[t]{2}{*}{ HRCT findings } & \multirow{2}{*}{$\begin{array}{l}\text { Disease extent } \\
\%\end{array}$} & \multicolumn{2}{|l|}{ KL-6 } & \multicolumn{2}{|l|}{ SP-D } \\
\hline & & $\mathrm{r}_{\mathrm{S}}$ & $\mathrm{p}$ value & $\mathrm{r}_{\mathrm{S}}$ & $\mathrm{p}$ value \\
\hline Ground-glass attenuation without traction bronchiectasis & $16.0 \pm 18.1$ & -0.010 & NS & 0.457 & $<0.05$ \\
\hline Airspace consolidation without traction bronchiectasis & $3.3 \pm 14.9$ & 0.278 & NS & -0.051 & NS \\
\hline Ground-glass attenuation with traction bronchiectasis & $16.6 \pm 13.5$ & 0.270 & NS & -0.517 & $<0.05$ \\
\hline Airspace consolidation with traction bronchiectasis & $4.4 \pm 5.6$ & -0.321 & NS & 0.292 & NS \\
\hline Honeycombing & $0.4 \pm 1.8$ & -0.074 & NS & 0.179 & NS \\
\hline Overall extent of interstitial disease & $40.8 \pm 17.5$ & 0.541 & $<0.01$ & 0.228 & NS \\
\hline Inflammatory pattern & $19.4 \pm 20.9$ & 0.211 & NS & 0.501 & $<0.05$ \\
\hline Fibrotic pattern & $21.4 \pm 11.6$ & 0.147 & NS & -0.460 & $<0.05$ \\
\hline
\end{tabular}

Disease extent is expressed as mean \pm SD. $r_{S}=$ Spearman's rank correlation between the extent of HRCT findings and serum marker levels. NS = Not significant. The inflammatory pattern was defined by the absence of traction bronchiectasis on ground-glass attenuation and consolidation. The fibrotic pattern was defined by the presence of traction bronchiectasis on ground-glass attenuation and consolidation, and honeycombing, as described in the Methods section.

Fig. 1. Relationship between baseline HRCT scores and serum biomarkers. a Baseline HRCT scores vs. serum levels of KL-6 $\left(\mathrm{r}_{\mathrm{S}}=0.453 ; \mathrm{p}<0.05\right)$. Statistical analysis was done by Spearman's rank correlation. b Baseline HRCT score vs. SP-D levels (correlation was not significant).
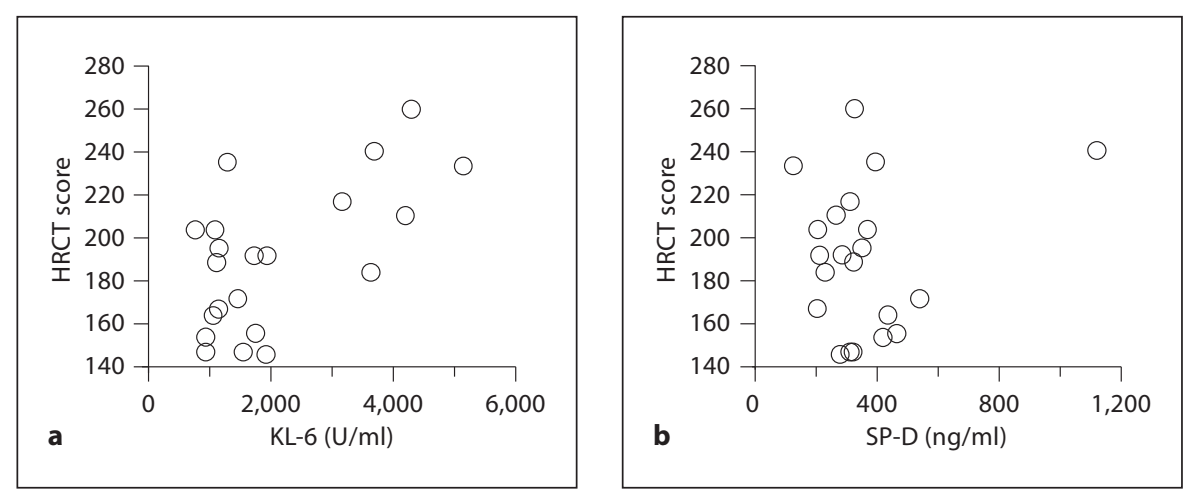

the total HRCT score showed a significant, but weak, positive correlation with serum KL-6 $\left(\mathrm{r}_{\mathrm{S}}=0.447, \mathrm{p}=0.0453\right.$, data not shown). However, no correlation was observed between the HRCT score and SP-D levels. In cellular NSIP patients $(n=3)$, there was no significant correlation between the HRCT score and KL-6 and SP-D levels. Because the number of patients with cellular NSIP was very small, we did not assess the association in each subtype. There was no correlation between KL-6 and SP-D levels.

\section{Associations between the 6 Types of HRCT Findings \\ for Disease Extent and Serum Levels of KL-6 and $S P-D$}

The HRCT findings and the correlation between the extent of each finding and the overall extent of interstitial disease and the serum levels of KL- 6 and SP-D are summarized in table 3. The area of ground-glass attenuation without traction bronchiectasis involved $16.0 \%$ of the lung parenchyma. The extent of the area showed a significant but weak positive correlation with the SP-D levels $\left(\mathrm{r}_{\mathrm{S}}=\right.$ 0.457; $\mathrm{p}<0.05)$. Ground-glass attenuation with traction bronchiectasis involved $16.6 \%$ of the lung parenchyma. However, the extent of this finding showed a significant negative correlation with the SP-D levels $\left(\mathrm{r}_{\mathrm{S}}=-0.517 ; \mathrm{p}<\right.$ 0.05 ). The extent of airspace consolidation without traction bronchiectasis, with traction bronchiectasis, or honeycombing involved $3.3,4.4$, and $0.4 \%$ of the lung parenchyma, respectively. There were no significant correlations between the extent of these findings and the serum levels of SP-D. Further, there was no correlation between the KL- 6 levels and any of the CT findings, whereas the overall extent of interstitial disease (total extent $=40.8 \pm$ $17.5 \%)$ was significantly correlated with the serum levels of KL-6 $\left(\mathrm{r}_{\mathrm{S}}=0.541 ; \mathrm{p}<0.01\right)$. These results indicate that unlike the serum levels of KL-6, those of SP-D may be associated with the presence of ground-glass attenuation 
with/without traction bronchiectasis, and KL-6 levels may reflect the overall extent of interstitial lesions in NSIP.

\section{Associations between the HRCT Patterns}

(Inflammatory or Fibrotic) and the Serum Levels of

$K L-6$ and $S P-D$

To determine if the serum levels of KL- 6 or SP-D were correlated to the histopathological conditions, we divided the HRCT findings into 2 patterns (inflammatory or fibrotic), as mentioned in the Methods. As shown in table 3 , there was a positive correlation between the extent of the inflammatory pattern and the serum level of SP-D $\left(r_{S}=0.501 ; p<0.05\right)$. In addition, the extent of the fibrotic pattern showed an inverse correlation with SP-D levels $\left(r_{S}=-0.460 ; p<0.05\right)$. Neither the inflammatory nor the fibrotic patterns showed significant correlations with the KL-6 levels. These results indicate that the serum levels of SP-D may be reflective of the relative amounts of inflammation and fibrosis in NSIP patients, although there was no correlation between the HRCT patterns (inflammatory or fibrotic) and the KL-6 and SP-D levels in each pathological subgroup.

\section{Changes in the Extent of HRCT Findings and Their}

Associations with the Serum Levels of KL-6 and SP-D before and after Treatment

Seventeen NSIP patients were examined to determine the relationship between the changes in the disease extent and the serum levels of KL- 6 or SP-D before and after treatment. The percent change from the baseline was determined at follow-up, and a positive change indicated improvement. After treatment, significant improvements were found (table 4) for the percent change in the total HRCT score (11.6 $\pm 14.2 \% ; \mathrm{p}=0.006)$, the overall extent of interstitial disease $(23.1 \pm 33.4 \%$; $p=0.023)$, and the serum levels of KL-6 (23.0 $\pm 28.8 \%$; $=0.004)$ and SP-D $(32.7 \pm 32.2 \% ; \mathrm{p}=0.002)$. The mean percent changes in extent of ground-glass attenuation without traction bronchiectasis, consolidation without traction bronchiectasis, ground-glass attenuation with traction bronchiectasis, consolidation with traction bronchiectasis, and honeycombing were $37.4 \pm 57.1,20.7 \pm 29.3,-14.3 \pm 83.3,71.4$ \pm 43.8 , and $-5.1 \pm 63.5 \%$, respectively. The mean percent change in the extent of the inflammatory pattern $(37.7 \pm 55.1 \%)$ was statistically significant $(\mathrm{p}=0.048)$.

As shown in figure 2, the change in the overall extent of interstitial disease on follow-up CT was significantly related to the change in the serum levels of KL-6 $\left(\mathrm{r}_{\mathrm{S}}=\right.$ $0.511 ; \mathrm{p}<0.05)$ and SP-D $\left(\mathrm{r}_{\mathrm{S}}=0.614 ; \mathrm{p}<0.01\right)$. Among the 2 HRCT patterns, the change in the extent of the fibrotic
Table 4. Percent changes of improvement in HRCT score, HRCT findings and serum maker levels

\begin{tabular}{lrl}
\hline & $\begin{array}{l}\text { Change } \\
\%\end{array}$ & $\begin{array}{l}\mathrm{p} \\
\text { value }\end{array}$ \\
\hline $\begin{array}{l}\text { Total HRCT score } \\
\text { Overall extent of interstitial disease }\end{array}$ & $11.6 \pm 14.2$ & 0.006 \\
$\begin{array}{l}\text { Ground-glass attenuation without } \\
\quad \text { traction bronchiectasis }\end{array}$ & $23.1 \pm 33.4$ & 0.023 \\
$\begin{array}{l}\text { Consolidation without } \\
\quad \text { traction bronchiectasis }\end{array}$ & & \\
$\begin{array}{l}\text { Ground-glass attenuation with } \\
\quad \text { traction bronchiectasis }\end{array}$ & $20.7 \pm 29.3$ & 0.333 \\
Consolidation with traction bronchiectasis & $-14.3 \pm 83.3$ & 0.601 \\
Honeycombing & $-5.1 \pm 63.5$ & 0.014 \\
Inflammatory pattern & $37.7 \pm 55.1$ & 0.521 \\
Fibrotic pattern & $14.5 \pm 46.1$ & 0.048 \\
KL-6 & $23.0 \pm 28.8$ & 0.004 \\
SP-D & $32.7 \pm 32.2$ & 0.002
\end{tabular}

The percent change from baseline was determined after treatment, and a positive percent change indicated improvement. Data are expressed as means $\pm \mathrm{SD}$.

Table 5. Correlation between the percent changes in HRCT patterns and serum marker levels

\begin{tabular}{|c|c|c|c|c|}
\hline \multirow[t]{2}{*}{ HRCT patterns } & \multicolumn{2}{|c|}{ KL- 6 change } & \multicolumn{2}{|c|}{ SP-D change } \\
\hline & $\mathrm{r}_{\mathrm{S}}$ & $\mathrm{p}$ value & $\mathrm{r}_{\mathrm{S}}$ & $\mathrm{p}$ value \\
\hline Inflammatory pattern change, $\%$ & 0.336 & NS & 0.125 & NS \\
\hline Fibrotic pattern change, $\%$ & 0.516 & $<0.05$ & 0.597 & $<0.05$ \\
\hline
\end{tabular}

See footnotes in table 3. $\mathrm{r}_{\mathrm{S}}=$ Spearman's rank correlation coefficient between the percent change in serum marker levels and that of the extent of each HRCT finding during the follow-up period.

pattern correlated significantly with the changes in the levels of KL- $6\left(r_{S}=0.516 ; p<0.05\right)$ and SP-D $\left(r_{S}=0.597\right.$; $\mathrm{p}<0.05$; table 5). In particular, the change in the extent of ground-glass attenuation with traction bronchiectasis exhibited a high correlation with the changes in the KL-6 levels $\left(\mathrm{r}_{\mathrm{S}}=0.695 ; \mathrm{p}<0.01\right.$, data not shown).

\section{Discussion}

In the present study, using a well-characterized group of patients with biopsy-proven NSIP, we showed that (1) the total HRCT score and the overall extent of interstitial disease were significantly correlated with the serum level 
Fig. 2. Relationship between percent changes in disease extent after treatment and serum biomarker levels. A change in each variable is defined as percent change from the baseline. A positive percent change indicates improvement. a Change in disease extent vs. change in KL- 6 levels $\left(r_{S}=\right.$ $0.511 ; \mathrm{p}<0.05)$. Statistical analysis was done by Spearman's rank correlation. b Change in disease extent vs. change in SP-D levels $\left(\mathrm{r}_{\mathrm{S}}=0.614 ; \mathrm{p}<0.01\right)$.
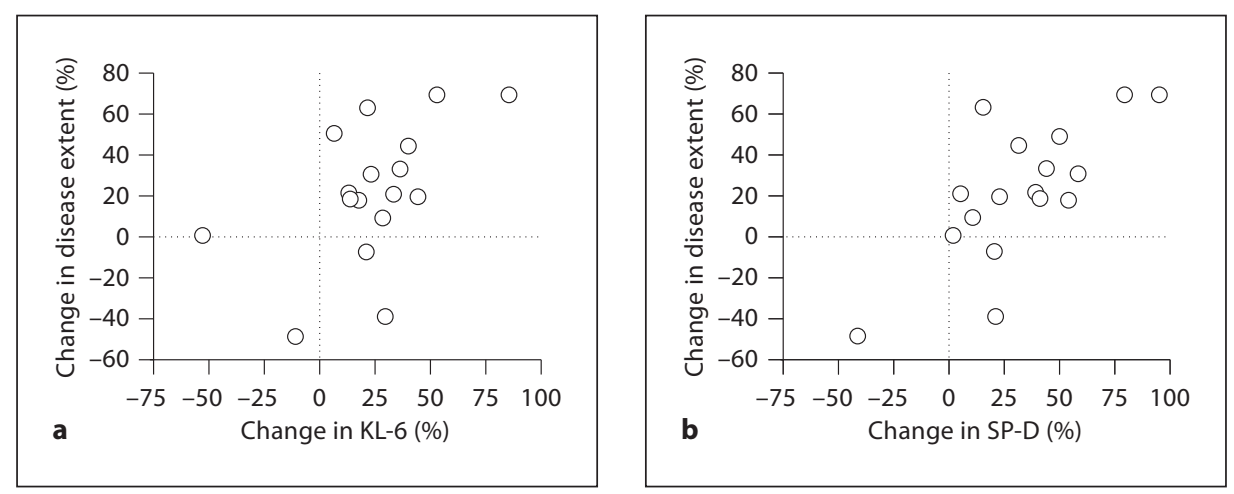

of KL- 6 at initial presentation, (2) the serum level of SP-D was positively correlated with the extent of ground-glass attenuation without traction bronchiectasis and the inflammatory pattern, (3) the serum level of SP-D was inversely correlated with the extent of ground-glass attenuation with traction bronchiectasis and the fibrotic pattern, and (4) a change in the disease extent during the CT follow-up period was reflected in the levels of both serum markers, especially of KL-6. HRCT imaging provides detailed information on the characteristics of a variety of lung inflammatory and fibrotic processes in interstitial lung disease; hence, our data indicate that the different results of the serum levels of KL-6 and SP-D may reflect the distinct HRCT patterns in NSIP.

KL- 6 and SP-D are lung-specific proteins secreted primarily by alveolar type II cells, and elevated levels of these serum marker proteins indicate activation of type II cells in the injured lung. Previous reports have suggested that these markers are reliable tools for the detection of various types of interstitial pneumonia, including IIPs such as IPF [12-14, 18-21]. However, it was not clear whether the serum levels of KL-6 and SP-D correlated with HRCT findings in NSIP.

NSIP, which is a subset of IIPs, is a recently described clinico-pathological entity [7]. According to the relative amounts of inflammation and fibrosis, NSIP is classified into 2 or 3 categories $[3,7,22]$. The results of few previous studies, which compared HRCT and pathological findings, suggest that the CT scans of NSIP patients have some characteristic features $[9,10]$. Based on the evaluation of 55 patients, Johkoh et al. [10] reported that ground-glass attenuation and consolidation, with a low degree of traction bronchiectasis and intralobular reticulation, were observed in patients with cellular NSIPs. They also showed that the HRCT findings of fibrotic NSIPs consist of ground-glass attenuation with extensive traction bronchiectasis, intralobular reticular opacities, and honeycombing. A recent study, in which morphological features associated with treatment outcome were identified by the assessment of serial HRCT findings, demonstrated that a predominant inflammatory pattern (ground-glass attenuation/consolidation) was associated with a better outcome than a predominant fibrotic pattern (reticulation/honeycombing), although the HRCT imaging was considerably heterogeneous [23]. On the basis of these correlations between HRCT findings and pathological features, we graded the HRCT findings in our study on a 6-point scale corresponding to the consecutive pathological phases, as described in the Methods section.

Although the serum levels of KL-6 did not show a significant correlation with individual HRCT findings, they were correlated with the total HRCT score and the overall extent of interstitial lesions in NSIP. Meanwhile, the serum levels of SP-D showed a significant but weak correlation with the extent of ground-glass attenuation without traction bronchiectasis and the area of the inflammatory pattern. There is no available information to account for the different behaviors of these serum markers in NSIP. On the basis of the findings of our study, we can interpret that the serum levels of KL-6 reflect the overall extent of the pathological stages of the lung parenchyma in NSIP patients, including both inflammatory and fibrotic lesions. In contrast, the serum levels of SP-D might mainly be reflective of the extent of inflammatory lesions. A previous study has shown that regenerative epithelial cells, which are the primary origin of KL-6 and SP-D, show different phenotypes in the less fibrous areas and the fibrotic areas in NSIP [24]. Ishii et al. [25] have reported that the levels of SP-D in the bronchoalveolar lavage fluid of patients with IPF were significantly lower than those of patients with NSIP. In our study, the serum levels of KL-6 
did not correlate with those of SP-D. Although there was no direct correlation between histological subgroups (cellular or fibrotic) of NSIP and the serum maker levels, the apparent discrepancy in the correlation between the HRCT findings and the KL- 6 and SP-D levels observed in our study may be attributed, in part, to the predominant histological status of the patients with NSIP.

Interestingly, SP-D levels were also inversely correlated with the extent of ground-glass attenuation with traction bronchiectasis and the area of the fibrotic pattern. Although ground-glass attenuation on HRCT scans is known to indicate the presence of pathological disease activity and a potentially treatable abnormality [8], the presence of traction bronchiectasis is commonly suggestive of the progression to fibrosis in NSIP as well as IPF and acute interstitial pneumonia $[10,11,20]$. Our results may suggest that the serum levels of SP-D correlate with the activity of NSIP but not with the progression of fibrosis.

In the present study, we evaluated serial levels of serum markers obtained in NSIP to clarify the association of the changes in HRCT findings, using the overall extent of disease and individual HRCT findings after corticosteroid and/or immunosuppressive therapy. Our data demonstrate that the percent changes in the serum levels of KL- 6 and SP-D after treatment were reflected in disease extent by CT. Other investigators have suggested that the changes in the serum biomarker levels are valuable indicators of disease activity and the subsequent therapeutic management in patients with interstitial lung disease [12, $13,18]$. Few studies on NSIP have shown that the extent of decrease on follow-up CT is well correlated with the improvement in pulmonary function, such as forced vital capacity and diffusing capacity of carbon monoxide [26]. In the present study, 13 patients (73\%) improved with $10 \%$ reduction in the overall extent and 2 patients (12\%) deteriorated with a $10 \%$ increase in the extent. Although the mean percent change in vital capacity after treatment was $8.3 \pm 14.6 \%$, there was no statistical association between response to treatment in terms of histological subtype, overall extent, HRCT score, serum markers, and percent VC. In addition, we showed that the percent change in the fibrotic HRCT finding, rather than the percent change in the inflammatory finding, correlated significantly with both KL-6 and SP-D levels. Furthermore, there was a good correlation between the response in terms of disease extent of ground-glass attenuation with traction bronchiectasis and decreased KL-6 levels. However, in comparison with a substantial improvement in the inflammatory pattern $(37.7 \pm 55.1 \%)$ after therapy, only minor im- provement in the fibrotic pattern $(14.6 \pm 46.1 \%)$ was observed. Since the area of ground-glass attenuation with traction bronchiectasis in NSIP has been shown to be a reliable indicator for the presence of fibrosis, the unexpected results obtained in the present study might be attributed to reversible fibrosis, as described previously [27].

We present some limitations of the present study. First, the number of patients with NSIP was not large; however, in all cases the diagnosis was histologically confirmed by surgical lung biopsy. Second, we evaluated the extent of HRCT findings by visual examinations. This subjective definition may not accurately reflect the extent of disease. Third, no information was collected for long-term follow-up. Whether the changes in the KL-6 and SP-D levels will reflect the HRCT findings and the subsequent long-term outcome remains to be clarified. Fourth, comprehensive analysis of the correlation between HRCT findings and serum levels of KL-6 and SP-D was not observed to distinguish between the histological subtypes of NSIP. Although surgical lung biopsy is the gold standard for the pathological diagnosis of NSIP, it is a highly invasive procedure for patients with interstitial pneumonia in clinical practice. In previous reports, the relationship between radiological findings and histopathology in NSIP has been well acknowledged. Therefore, we attempted to examine whether the serum levels of KL-6 and SP-D in NSIP could reflect the inflammatory and/or fibrotic changes disclosed by HRCT scans. Because there was no correlation between histological subtypes and serum marker levels, further studies will be required to validate the clinical values of KL- 6 and SP-D during the inflammatory/fibrotic process in a larger cohort of NSIP patients.

In conclusion, our results suggest that in NSIP the serum level of KL-6 correlated with the overall extent of interstitial disease and ground-glass attenuation, and that the serum level of SP-D correlated positively with the area of ground-glass attenuation without traction bronchiectasis and the inflammatory pattern on HRCT, although there was no information on the correlation between histological findings and serum marker levels. Further, the decrease in disease extent after treatment was reflected in the decreased levels of both serum markers, especially of KL-6. A prospective study including serial examinations is needed to determine whether these biomarkers are associated with clinical parameters such as HRCT findings. 


\section{Acknowledgments}

We would like to thank Drs. Thomas V. Colby, Masanori Kitaichi, and Yuh Fukuda for establishing the pathological diagnoses.

\section{Financial Disclosure and Conflicts of Interest}

None of the authors has any conflict of interest associated with this study.

\section{References}

1 American Thoracic Society/European Respiratory Society: American Thoracic Society/European Respiratory Society International Multidisciplinary Consensus Classification of the Idiopathic Interstitial Pneumonias. This joint statement of the American Thoracic Society (ATS), and the European Respiratory Society (ERS) was adopted by the ATS board of directors, June 2001 and by the ERS Executive Committee, June 2001. Am J Respir Crit Care Med 2002;165:277-304.

-2 Nicholson AG, Colby TV, du Bois RM, Hansell DM, Wells AU: The prognostic significance of the histologic pattern of interstitial pneumonia in patients presenting with the clinical entity of cryptogenic fibrosing alveolitis. Am J Respir Crit Care Med 2000; 162:2213-2217.

-3 Travis WD, Matsui K, Moss J, Ferrans VJ: Idiopathic nonspecific interstitial pneumonia: prognostic significance of cellular and fibrosing patterns: survival comparison with usual interstitial pneumonia and desquamative interstitial pneumonia. Am J Surg Pathol 2000;24:19-33.

-4 Kim DS, Collard HR, King TE Jr: Classification and natural history of the idiopathic interstitial pneumonias. Proc Am Thorac Soc 2006;3:285-292.

-5 Travis WD, Hunninghake G, King TE Jr, Lynch DA, Colby TV, Galvin JR, Brown KK, Chung MP, Cordier JF, du Bois RM, Flaherty KR, Franks TJ, Hansell DM, Hartman TE, Kazerooni EA, Kim DS, Kitaichi M, Koyama T, Martinez FJ, Nagai S, Midthun DE, Müller NL, Nicholson AG, Raghu G, Selman M, Wells A: Idiopathic nonspecific interstitial pneumonia: report of an American Thoracic Society project. Am J Respir Crit Care Med 2008;177:1338-1347.

-6 Quadrelli S, Molinari L, Ciallella L, Spina JC, Sobrino E, Chertcoff J: Radiological versus histopathological diagnosis of usual interstitial pneumonia in the clinical practice: does it have any survival difference? Respiration 2010;79:32-37.

7 Katzenstein AL, Fiorelli RF: Nonspecific interstitial pneumonia/fibrosis. Histologic features and clinical significance. Am J Surg Pathol 1994;18:136-147.

-8 Leung AN, Miller RR, Muller NL: Parenchymal opacification in chronic infiltrative lung diseases: CT-pathologic correlation. Radiology 1993;188:209-214.
9 Kim TS, Lee KS, Chung MP, Han J, Park JS, Hwang JH, Kwon OJ, Rhee CH: Nonspecific interstitial pneumonia with fibrosis: highresolution $\mathrm{CT}$ and pathologic findings. Am I Roentgenol 1998;171:1645-1650.

10 Johkoh T, Müller NL, Colby TV, Ichikado K, Taniguchi H, Kondoh Y, Fujimoto K, Kinoshita M, Arakawa H, Yamada H, Suga M, Ando M, Koyama M, Nakamura H: Nonspecific interstitial pneumonia: correlation between thin-section CT findings and pathologic subgroups in 55 patients. Radiology 2002;225:199-204.

11 Wittram C, Mark EJ, McLoud TC: CT-histologic correlation of the ATS/ERS 2002 classification of idiopathic interstitial pneumonias. Radiographics 2003;23:1057-1071.

12 Kohno N, Kyoizumi S, Awaya Y, Fukuhara $H$, Yamakido M, Akiyama M: New serum indicator of interstitial pneumonitis activity. Sialylated carbohydrate antigen KL-6. Chest 1989;96:68-73.

13 Takahashi H, Kuroki Y, Tanaka H, Saito T, Kurokawa K, Chiba H, Sagawa A, Nagae H, Abe S: Serum levels of surfactant proteins A and $\mathrm{D}$ are useful biomarkers for interstitial lung disease in patients with progressive systemic sclerosis. Am J Respir Crit Care Med 2000;162:258-263.

14 Greene KE, King TE Jr, Kuroki Y, BucherBartelson B, Hunninghake GW, Newman LS, Nagae H, Mason RJ: Serum surfactant proteins-A and -D as biomarkers in idiopathic pulmonary fibrosis. Eur Respir $\mathrm{2002}$; 19:439-446.

15 Ohnishi H, Yokoyama A, Kondo K, Hamada $\mathrm{H}$, Abe M, Nishimura K, Hiwada K, Kohno N: Comparative study of KL-6, surfactant protein-A, surfactant protein-D, and monocyte chemoattractant protein-1 as serum markers for interstitial lung diseases. Am J Respir Crit Care Med 2002;165:378-381.

16 Sakamoto K, Taniguchi H, Kondoh Y, Johkoh T, Sumikawa H, Kimura T, Nishiyama O, Kato K, Kataoka K, Ono K, Kitaichi M, Hasegawa Y: Serum KL-6 in fibrotic NSIP: correlations with physiologic and radiologic parameters. Respir Med 2010;104:127-133.

17 Okada F, Ando Y, Honda K, Tanoue S, Matsumoto S, Mori H: Comparison of pulmonary CT findings and serum KL-6 levels in patients with cryptogenic organizing pneumonia. Br J Radiol 2009;82:212-218.
18 Kohno N: Serum marker KL-6/MUC1 for the diagnosis and management of interstitial pneumonitis. J Med Invest 1999;46:151-158.

19 Hirasawa Y, Kohno N, Yokoyama A, Inoue Y, Abe M, Hiwada K: KL-6, a human MUC1 mucin, is chemotactic for human fibroblasts. Am J Respir Cell Mol Biol 1997;17:501-507.

20 Ichikado K, Suga M, Müller NL, Taniguchi H, Kondoh Y, Akira M, Johkoh T, Mihara N, Nakamura H, Takahashi M, Ando M: Acute interstitial pneumonia: comparison of highresolution computed tomography findings between survivors and nonsurvivors. Am J Respir Crit Care Med 2002;165:1551-1556.

21 Yokoyama A, Kondo K, Nakajima M, Matsushima T, Takahashi T, Nishimura M, Bando M, Sugiyama Y, Totani Y, Ishizaki T, Ichiyasu H, Suga M, Hamada H, Kohno N: Prognostic value of circulating KL-6 in idiopathic pulmonary fibrosis. Respirology 2006;11:164168.

22 Nagai S, Kitaichi M, Itoh H, Nishimura K, Izumi T, Colby TV: Idiopathic nonspecific interstitial pneumonia/fibrosis: comparison with idiopathic pulmonary fibrosis and BOOP. Eur Respir J 1998;12:1010-1019.

23 Screaton NJ, Hiorns MP, Lee KS, Franquet T, Johkoh T, Fujimoto K, Ichikado K, Colby TV, Müller NL: Serial high resolution CT in nonspecific interstitial pneumonia: prognostic value of the initial pattern. Clin Radiol 2005; 60:96-104.

24 Hinata N, Takemura T, Ikushima S, Yanagawa T, Ando T, Okada J, Oritsu M, Koike M: Phenotype of regenerative epithelium in idiopathic interstitial pneumonias. J Med Dent Sci 2003;50:213-224.

25 Ishii H, Mukae H, Kadota J, Kaida H, Nagata T, Abe K, Matsukura S, Kohno S: High serum concentrations of surfactant protein A in usual interstitial pneumonia compared with non-specific interstitial pneumonia. Thorax 2003;58:52-57.

26 Kim EY, Lee KS, Chung MP, Kwon OJ, Kim TS, Hwang JH: Nonspecific interstitial pneumonia with fibrosis: serial high-resolution CT findings with functional correlation. AJR Am J Roentgenol 1999;173:949953.

27 Nishiyama O, Kondoh Y, Taniguchi H, Yamaki K, Suzuki R, Yokoi T, Takagi K: Serial high resolution CT findings in nonspecific interstitial pneumonia/fibrosis. J Comput Assist Tomogr 2000;24:41-46. 